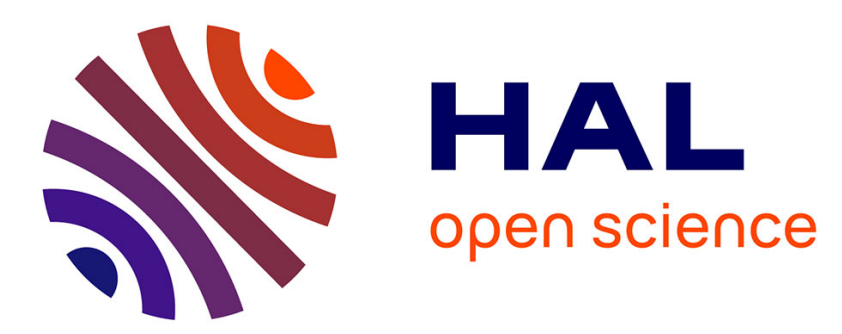

\title{
Aberration-accounting calibration for 3D single-molecule localization microscopy
}

Clément Cabriel, Nicolas Bourg, Guillaume Dupuis, Sandrine Leveque-Fort

\section{To cite this version:}

Clément Cabriel, Nicolas Bourg, Guillaume Dupuis, Sandrine Leveque-Fort. Aberration-accounting calibration for 3D single-molecule localization microscopy. Optics Letters, 2018, 43 (2), pp.174. 10.1364/OL.43.000174. hal-02322808

\section{HAL Id: hal-02322808 \\ https://hal.science/hal-02322808}

Submitted on 9 Dec 2019

HAL is a multi-disciplinary open access archive for the deposit and dissemination of scientific research documents, whether they are published or not. The documents may come from teaching and research institutions in France or abroad, or from public or private research centers.
L'archive ouverte pluridisciplinaire HAL, est destinée au dépôt et à la diffusion de documents scientifiques de niveau recherche, publiés ou non, émanant des établissements d'enseignement et de recherche français ou étrangers, des laboratoires publics ou privés. 
archives-ouvertes

\section{Aberration-accounting calibration for 3D single-molecule localization microscopy}

Clément Cabriel, Nicolas Bourg, Guillaume Dupuis, Sandrine Leveque-Fort

\section{To cite this version:}

Clément Cabriel, Nicolas Bourg, Guillaume Dupuis, Sandrine Leveque-Fort. Aberration-accounting calibration for 3D single-molecule localization microscopy. Optics Letters, Optical Society of America, 2018, 43 (2), pp.174. 10.1364/OL.43.000174 . hal-02322808

\section{HAL Id: hal-02322808 \\ https://hal.archives-ouvertes.fr/hal-02322808}

Submitted on 9 Dec 2019

HAL is a multi-disciplinary open access archive for the deposit and dissemination of scientific research documents, whether they are published or not. The documents may come from teaching and research institutions in France or abroad, or from public or private research centers.
L'archive ouverte pluridisciplinaire HAL, est destinée au dépôt et à la diffusion de documents scientifiques de niveau recherche, publiés ou non, émanant des établissements d'enseignement et de recherche français ou étrangers, des laboratoires publics ou privés. 


\title{
Aberration-accounting calibration for 3D single-molecule localization microscopy
}

\author{
Clément Cabriel, ${ }^{1, *}$ Nicolas Bourg, ${ }^{1}$ Guillaume Dupuis, ${ }^{2}$ and Sandrine Lévêque-Fort ${ }^{1,3}$ \\ ${ }^{1}$ Institut des Sciences Moléculaires d’Orsay (ISMO), CNRS, Univ. Paris Sud, Université Paris Saclay, bâtiment 520, rue André Rivière, \\ 91405 Orsay Cedex, France \\ ${ }^{2}$ Centre de Photonique BioMédicale (CPBM), CNRS, Fédération LUMAT, Univ. Paris Sud, Université Paris Saclay, bâtiment 520, rue André Rivière, \\ 91405 Orsay Cedex, France \\ ${ }^{3}$ e-mail: sandrine.leveque-fort@u-psud.fr \\ *Corresponding author: clement.cabrie/@u-psud.fr
}

Received 13 October 2017; revised 1 December 2017; accepted 3 December 2017; posted 4 December 2017 (Doc. ID 309151); published 3 January 2018

\begin{abstract}
We propose a straightforward sample-based technique to calibrate the axial detection in 3D single-molecule localization microscopy. Using microspheres coated with fluorescent molecules, the calibration curves of point spread function-shaping or intensity-based measurements can be obtained over the imaging depth range. This experimental method takes into account the effect of the spherical aberration without requiring computational correction. We demonstrate its efficiency for astigmatic imaging in a $1.2 \mu \mathrm{m}$ range above the coverslip. () 2018 Optical Society of America
\end{abstract}

OCIS codes: (180.2520) Fluorescence microscopy; (180.6900) Three-dimensional microscopy; (120.0120) Instrumentation, measurement, and metrology.

https://doi.org/10.1364/OL.43.000174

Single-molecule localization microscopy (SMLM) is now a well-established method used in biology for a wide range of applications, especially single particle tracking [1] in living samples and super-resolution structural observation using (f) PALM, (d)STORM, or PAINT [2-6]. Although retrieving the lateral positions in the focus plane is quite straightforward, $3 \mathrm{D}$ detection requires complementary axial information that can be provided either by point spread function (PSF) shape measurement methods [7-11], in which the axial information is encoded in the shape of the spots, or by intensity-based methods (such as interferometric measurements [12,13] or supercritical angle fluorescence (SAF) detection [14,15]), which rely on the dependence of the intensity on the depth.

In most cases, such an axial detection scheme requires a calibration to know the relationship between the measured value and the depth, or at least an experimental verification to validate the consistency of the results obtained from a theoretical characteristic curve. Most of the time this is performed by using fluorescent beads or molecules deposited on a coverslip and scanning the objective with a piezoelectric stage to introduce defocus in the system. While inexpensive and simple to perform, this method exhibits several drawbacks arising from the refractive index mismatch between the sample and the glass coverslip.

First, the distance over which the focus plane is moved is not equal to the displacement of the objective. In practice, this socalled focal shift effect produces a stretching of the apparent distances. Although theoretical formulas [16] and experimental protocols $[17,18]$ of various complexities are available to determine the value of the correction factor for different depth ranges, these methods are not sufficient to provide readily usable calibration data suitable for SMLM experiments.

Indeed, they do not account for the effect of the spherical aberration on the PSFs. Such an aberration alters the shape of the spots and, thus, induces a bias in the axial positions detected through PSF-shaping methods. Calibrations performed by scanning the objective do not allow one to record the PSFs corresponding to a realistic experimental situation where the focus plane is fixed. Several techniques have been proposed to circumvent this issue, notably numerical computation [19]. While this technique does not require a cumbersome experimental procedure, it does not fully correct the effect of the spherical aberration. Deng and Shaevitz proposed a reliable experimental method using optical tweezers to axially move fluorescent beads relative to the object plane [20] at the cost of a major modification of the setup. Similarly, adaptive optics can be used to correct the spherical aberration [21], but this requires expensive devices and induces a loss of photons.

We designed a fully experimental, sample-based calibration method to provide unbiased calibration results that can be used for 3D SMLM measurements. In this protocol, all the axial information needed is provided by the known geometry of the sample, and the acquisitions are performed in the nominal conditions, i.e., for a given objective at a given position and for a given sample refractive index and fluorophore emission wavelength.

More specifically, we used $15.0 \mu \mathrm{m}( \pm 1.5 \mu \mathrm{m})$ diameter latex microspheres coated with biotin (Kisker Biotech, PC-BX15.0), on which we attached the fluorophores of interest, 
namely Alexa Fluor (AF) 647 functionalized with streptavidin (Life Technologies, S21374). The sample was elaborated as follows: we prepared a solution containing $500 \mu \mathrm{L}$ of water, $500 \mu \mathrm{L}$ of PBS, $100 \mu \mathrm{L}$ of microsphere solution, and $0.5 \mu \mathrm{L}$ of streptavidin-functionalized AF647. This solution was centrifuged $20 \mathrm{~min}$ at $13.4 \mathrm{krpm}$. The liquid was then removed and replaced with $100 \mu \mathrm{L}$ of PBS. By vortexing the aliquot, the deposit was dissolved. We then took $50 \mu \mathrm{L}$ of the final solution, delicately laid it on a glass coverslip and waited for $20 \mathrm{~min}$ after covering it to protect it from light and evaporation. Finally, we gently added $500 \mu \mathrm{L}$ of imaging dSTORM buffer (dSTORM smart kit, Abbelight). A similar sample was used in [18], but only in confocal microscopy to measure the focal shift. The interest of such a sample relies on the fact that, once the lateral position of the center $\left(x_{0}, y_{0}\right)$ and the radius $R$ of each microsphere are known, the depth of any molecule can be calculated from its measured lateral position $(x, y)$ using the following equation [Fig. 1(a)]:

$$
\rho^{2}+(R-z)^{2}=R^{2},
$$

where $\rho=\sqrt{\left(x-x_{0}\right)^{2}+\left(y-y_{0}\right)^{2}}$ is the lateral radial position to the center of the microsphere. This directly gives the height $z$ of the molecule assuming that only the lower part of the microsphere is imaged (which is reasonable since $R$ is typically much higher than the depth of field of the system):

$$
z=R-\sqrt{R^{2}-\rho^{2}} \text {. }
$$

The sample was observed using a standard homemade superlocalization microscope. The coverslip was illuminated with a $637 \mathrm{~nm}$ laser (Obis 637LX, $140 \mathrm{~mW}$, Coherent). Our setup included a Nikon Eclipse $\mathrm{Ti}$ inverted microscope with a Nikon Perfect Focus System, and a Nikon Apo TIRF 100x NA1.49 objective. The camera was a $512 \times 512$ - pixel electron-multiplying charge-coupled device (EMCCD) (Andor, iXon3), with one pixel corresponding to $100 \mathrm{~nm}$ in the object plane. We first acquired a diffraction-limited image of the sample at low illumination power $\left(0.05 \mathrm{~kW} \cdot \mathrm{cm}^{-2}\right)$ in the equatorial plane of the sphere, i.e., at $R$ above the coverslip [Fig. 1(b)], which was used to measure both the radius $R$ and the center $\left(x_{0}, y_{0}\right)$. The effect of the diffraction was taken into account in this measurement.

We first performed an acquisition with the DONALD 3D super-resolution technique [14], which provides absolute axial information based on the SAF intensity measurement [22]. As this detection uses a characteristic curve obtained from a theoretical model, it does not require experimental calibration. Thus, it provides a reliable method to verify the sample shape. As seen on the $\rho-z$ profile [Fig. 1(c)], the microspheres exhibit the expected curvature in the probed area. In particular, they do not seem to significantly flatten out at the contact point with the coverslip.

Having verified the validity of the geometry of the sample, we carried out acquisitions to obtain the calibration curve of an astigmatism-based PSF-shaping detection scheme. The aberration was created by a cylindrical lens added in the detection path with a focal length and a position calculated to optimize the axial localization precision [23], giving a spacing of approximately $800 \mathrm{~nm}$ between the two focal lines. The focus (average point between the two focal lines) was set at $400 \mathrm{~nm}$ beyond the coverslip. We then increased the laser excitation power $\left(4 \mathrm{~kW} \cdot \mathrm{cm}^{-2}\right)$ to achieve a sufficient molecule density per

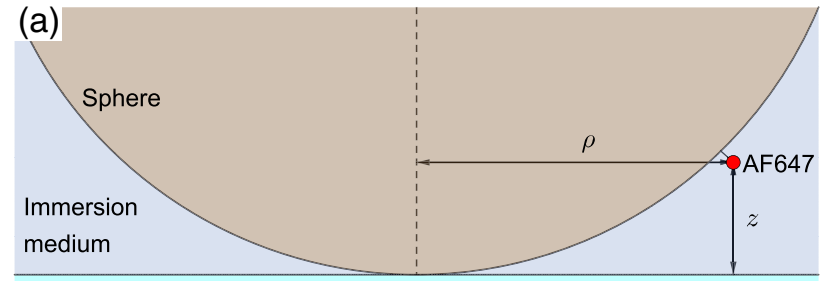

Coverslip
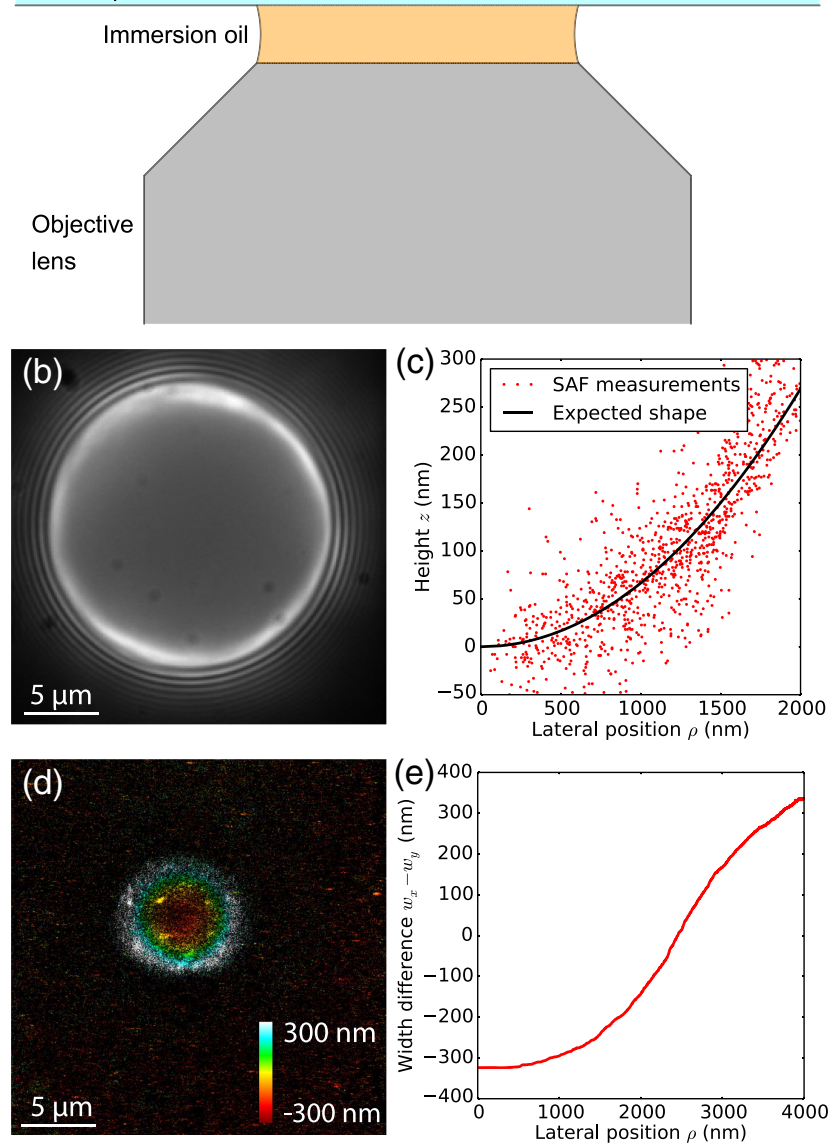

Fig. 1. Principle of the calibration protocol. (a) Schematic of the sample of coated microspheres deposited on a coverslip. (b) Raw diffraction limited image of a microsphere acquired in its equatorial plane (used to determine the radius and the center of the microsphere). (c) Depth profile detected with DONALD. The red circles correspond to the experimental data, and the black line stands for the curvature expected for a sphere of the measured radius. (d) Color-coded map of the width difference $\Delta w=w_{x}-w_{y}$ of the PSFs induced by the astigmatism. Note that the radius appears lower than in (b) since the depth of imaging limits the observation to a small portion of the sphere near the contact point with the coverslip. (e) Width difference $\Delta w$ profile as a function of the lateral position obtained from the sphere displayed in $(\mathrm{d})$.

frame (typically one molecule per $4 \mu \mathrm{m}^{2}$ per frame). The dSTORM data were acquired with $50 \mathrm{~ms}$ exposure time and 150 EMCCD gain and processed using a home-written Python code to extract the $x$ and $y$ positions of each spot, as well as their widths $w_{x}$ and $w_{y}$, via a Gaussian fitting. Both the lateral and axial drifts were corrected using a cross-correlation algorithm, and the lateral deformation of the image induced by the astigmatism was accounted for. 
The corresponding width difference map and lateral profile are presented in Figs. 1(d) and 1(e), respectively. Using $x, y, x_{0}, y_{0}$, and $R$, we determined the value of the depth $z$ of each molecule, which allowed us to plot the calibration curve $\left(w_{x}\right.$, $w_{y}$ ) as a function of $z$. For the statistical pooling of the localization data, we analyzed three different microspheres for a total of approximately 20.000 localization events. To reduce the influence of statistical uncertainties, the data were divided in $40 \mathrm{~nm}$ depth slices (using a sliding window pooling), each slice containing between 100 and 1.000 localized points.

As a comparison, we also conducted an acquisition on a sample of $20 \mathrm{~nm}$ fluorescent beads deposited on a glass coverslip (Invitrogen, F8783). The object plane was scanned through the sample by moving the objective by $25 \mathrm{~nm}$ steps, thanks to a piezoelectric stage, and the axial positions were corrected in order to take into account the focal shift. For each position of the objective, 25 fluorescent beads were imaged over 200 frames to reduce the statistical fluctuations by averaging. In order to compare the two methods, this calibration curve was translated using a common reference: when the focus is set $400 \mathrm{~nm}$ deep in the sample, the aberrations are the same for the $20 \mathrm{~nm}$ fluorescent beads as for fluorophores at $z=10 \mathrm{~nm}$ with the calibration technique using the labeled microspheres. Thus, the abscissa was shifted so that the curve overlaps with the microspheres calibration curve at $10 \mathrm{~nm}$ depth, corresponding to the same experimental configuration.

Thanks to the previously described method, we obtained the calibration curve of the astigmatism and compared it to the method using fluorescent beads deposited on a coverslip [Fig. 2(a)], which yields significantly different results. Note that because the experiment was performed in the nominal conditions, a single acquisition provides a calibration valid over the whole axial detection range of the instrument, namely $1.2 \mu \mathrm{m}$. The difference between the two methods is mainly due to the influence of the spherical aberration arising from the index mismatch on the PSF shapes, which is not considered in the latter case. The resulting axial bias [Fig. 2(b)] proves to behave in a very nonlinear way (in particular, it is either positive or negative depending on the depth), reaching values as large as $200 \mathrm{~nm}$ over a $1200 \mathrm{~nm}$ range. Such a result is in agreement with previous studies quantifying the effect of the spherical aberration in calibration experiments $[19,20,24]$, in terms of both curve shape and magnitude. To illustrate the importance of this difference, we performed measurements on a COS-7 sample labeled with AF647-phalloidin to image the actin network [Figs. 2(c) and 2(d)]. The slice profile presented in Fig. 2(e) highlights the axial bias that arises from the effect of the spherical aberration when using fluorescent beads immobilized on a coverslip to perform the calibration.

One of the key features of our technique is its precision, which makes it especially suitable for super-localization experiments. The statistical uncertainty $\sigma_{z}$ on the value of $z$ calculated from Eq. (2) decreases as $R$ increases. Indeed, the lower the local slope of the sphere surface, the lower the influence of the lateral localization precision. As it is necessary to image a whole microsphere to measure its radius and center, the optimal microsphere diameter one should choose to achieve the best precision is equal to the size of the field of view. Assuming that the center and the radius can be estimated with precisions around $50 \mathrm{~nm}$, a $15 \mu \mathrm{m}$ diameter microsphere yields axial precisions $\sigma_{z}$ down to $15 \mathrm{~nm}$ over a $1.2 \mu \mathrm{m}$ axial range. It should be noted that since the number of localized molecules on the microsphere is around 20.000 , the localization precision
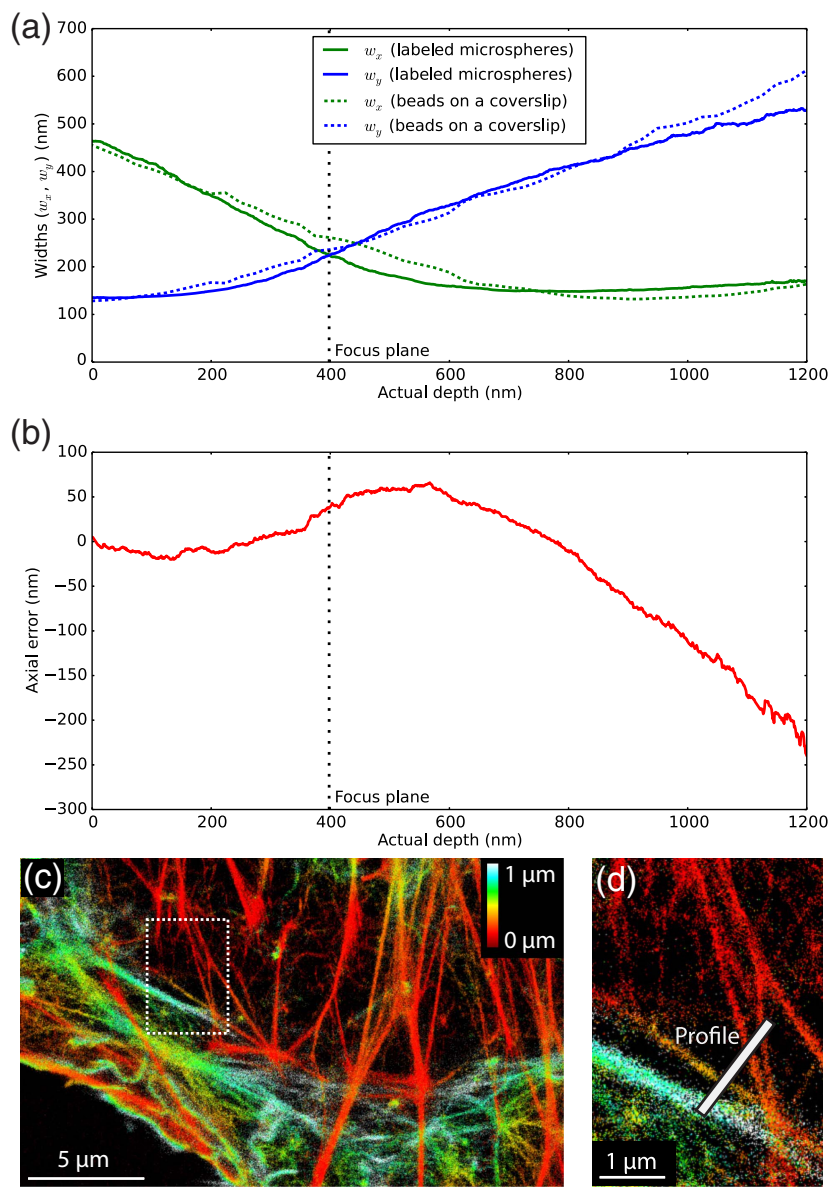

(e)

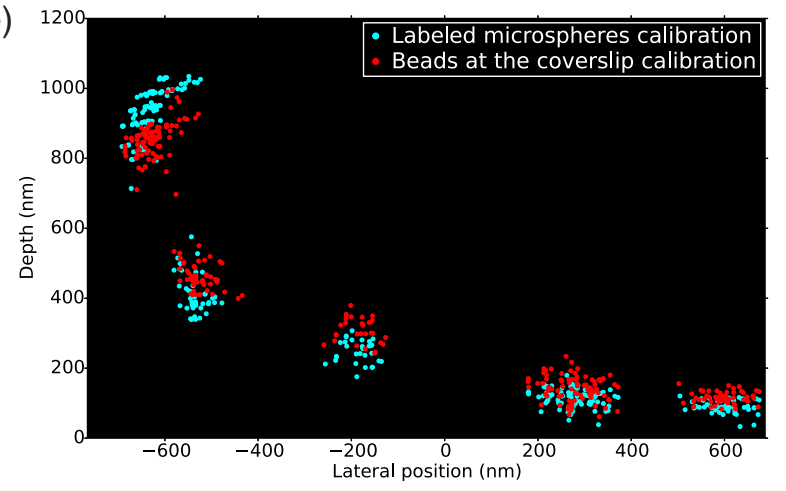

Fig. 2. Calibration results. (a) Calibration curve showing the widths $w_{x}$ and $w_{y}$ obtained by analyzing the data acquired from the labeled microsphere sample as a function of the distance to the coverslip. The comparison with the method using fluorescent beads at the coverslip is also displayed. The abscissa of the latter calibration method was translated so that the two curves coincide at $10 \mathrm{~nm}$ depth with a focus at $400 \mathrm{~nm}$ (thus corresponding to the same experimental configuration). (b) Error made on the measured axial position when using immobilized fluorescent beads on a coverslip to perform the calibration, showing the difference between the two calibration curves displayed in (a). (c) 3D (color-coded depth) localization image of actin labeled with AF647. (d) Zoom-in on the boxed region shown in (c). (e) $x-z$ profile of actin filaments along the axis displayed in (d), comparing the two calibration methods. 
has a much lower influence on the value of $\sigma_{z}$ than any error on the measurement of the radius or the center of the sphere.

We have demonstrated a fully experimental unbiased method to obtain the calibration curve of any PSF-shapingbased axial detection scheme in 3D SMLM. While the method does not require any computation, device interfacing, or modification of the optical setup, it accounts for both the focal shift and the effect of the spherical aberration on the PSFs by performing acquisitions in the nominal experimental conditions on a controlled geometry sample. These features make it suitable for a broad range of uses in SMLM as it could be used in (d)STORM, (f)PALM, and (DNA-)PAINT. Aside from the calibration of PSF-shaping methods, this technique could also be used as a means of experimental verification of the performances of 3D SMLM techniques requiring no calibration. Moreover, as the use of the geometrical relation giving the axial position from the lateral information is not specific to single-molecule experiments, the same type of sample could be used at wider depth ranges (up to several tens or hundreds of microns) with other imaging modalities, such as lightsheet microscopy, structured illumination, two-photon excitation or stimulated emission depletion microscopy, for instance to calibrate the axial positioning. In single-molecule imaging, however, the method could be further improved by labeling the microspheres with several dyes to perform a calibration at different emission wavelengths (either sequentially or simultaneously), thus getting rid of the chromatic aberration influence, a major bottleneck for multiple color/species 3D SMLM imaging.

Funding. Région Île-de-France (NanoSAF project); Centre National de la Recherche Scientifique (CNRS) (Mission Interdisciplinaire).

Disclosure. N. Bourg and S. Lévêque-Fort are shareholders in Abbelight.

\section{REFERENCES}

1. J. Deich, E. M. Judd, H. H. McAdams, and W. E. Moerner, Proc. Natl. Acad. Sci. USA 101, 15921 (2004).
2. E. Betzig, G. H. Patterson, R. Sougrat, O. W. Lindwasser, S. Olenych, J. S. Bonifacino, M. W. Davidson, J. Lippincott-Schwartz, and H. F. Hess, Science 313, 1642 (2006).

3. S. T. Hess, T. P. K. Girirajan, and M. D. Mason, Biophys. J. 91, 4258 (2006).

4. M. J. Rust, M. Bates, and X. Zhuang, Nat. Methods 3, 793 (2006).

5. S. van de Linde, A. Löschberger, T. Klein, M. Heidbreder, S. Wolter, M. Heilemann, and M. Sauer, Nat. Protoc. 6, 991 (2011).

6. A. Sharonov and R. M. Hochstrasser, Proc. Natl. Acad. Sci. USA 103 18911 (2006).

7. B. Huang, W. Wang, M. Bates, and X. Zhuang, Science 319, 810 (2008).

8. S. R. P. Pavani, M. A. Thompson, J. S. Biteen, S. J. Lord, N. Liu, R. J. Twieg, R. Piestun, and W. E. Moerner, Proc. Natl. Acad. Sci. USA 106, 2995 (2009).

9. Y. Shechtman, L. E. Weiss, A. S. Backer, S. J. Sahl, and W. E. Moerner, Nano Lett. 15, 4194 (2015).

10. M. F. Juette, T. J. Gould, M. D. Lessard, M. J. Mlodzianoski, B. S. Nagpure, B. T. Bennett, S. T. Hess, and J. Bewersdorf, Nat. Methods 5, 527 (2008).

11. C. Franke, M. Sauer, and S. van de Linde, Nat. Methods 14, 41 (2016).

12. G. Shtengel, J. A. Galbraith, C. G. Galbraith, J. Lippincott-Schwartz, J. M. Gillette, S. Manley, R. Sougrat, C. M. Waterman, P. Kanchanawong, M. W. Davidson, R. D. Fetter, and H. F. Hess, Proc. Natl. Acad. Sci. USA 106, 3125 (2009).

13. D. Aquino, A. Schönle, C. Geisler, C. V. Middendorff, C. A. Wurm, Y. Okamura, T. Lang, S. W. Hell, and A. Egner, Nat. Methods 8, 353 (2011).

14. N. Bourg, C. Mayet, G. Dupuis, T. Barroca, P. Bon, S. Lécart, E. Fort, and S. Lévêque-Fort, Nat. Photonics 9, 587 (2015).

15. J. Deschamps, M. Mund, and J. Ries, Opt. Express 22, 29081 (2014).

16. C. J. R. Sheppard and P. Török, J. Microsc. 185, 366 (1997).

17. A. Diaspro, F. Federici, and M. Robello, Appl. Opt. 41, 685 (2002).

18. B. P. Bratton and J. W. Shaevitz, PLoS ONE 10, e0134616 (2015).

19. R. McGorty, J. Schnitzbauer, W. Zhang, and B. Huang, Opt. Lett. 39, 275 (2014).

20. Y. Deng and J. W. Shaevitz, Appl. Opt. 48, 1886 (2009).

21. I. Izeddin, M. El Beheiry, J. Andilla, D. Ciepielewski, X. Darzacq, and M. Dahan, Opt. Express 20, 4957 (2012).

22. T. Barroca, K. Balaa, S. Lévêque-Fort, and E. Fort, Phys. Rev. Lett. 108, 218101 (2012).

23. B. Rieger and S. Stallinga, ChemPhysChem 15, 664 (2014).

24. S. Proppert, S. Wolter, T. Holm, T. Klein, S. van de Linde, and M. Sauer, Opt. Express 22, 10304 (2014). 